Urs Steiner

Am 31. Juli 1986 wurde die SAHP von Martin Furlenmeier (verstorben am 23.6.1994), Susanne Furlenmeier (verstorben am 6.4.2011), Clemens Dietrich und vom Autor, Urs Steiner, in Wohlen/AG gegründet. Die Absicht war eine möglichst breite, auch physikalische, chemische und botanische Wissensvermittlung der homöopathischen Heilmethode. Eine treibende Kraft war auch die Neugier auf das Unbekannte und die Suche nach den Zusammenhängen. Es dürfte uns gelungen sein, viele solcher Zusammenhänge aufgezeigt zu haben.

Wir betrachten den Patienten immer in seiner Ganzheit: Wir schauen bei Krankheit auf das Eigenheitliche [1], also das Pathognomonische, und gleichzeitig auf das Ungewöhnliche (oder Seltene) und Sonderliche (oder Unterschiedliche bei gleicher Krankheitsgattung). Bönninghausen gehörte $\mathrm{zu}$ den Ersten, der auch beim Homoion, also bei der Arznei, auf gleiche Weise vorging, indem er die Skaleninvarianz ermittelte, d.h. indem er all jene Symptome im Genius der einen Arznei begrifflich zusammenfasste, die sich auf allen Ebenen wiederholten. Er brachte auf den Nenner, was auffällt. Hering und Farrington [2] ergänzten den Genius der einzelnen Arznei noch mit dem Ungewöhnlichen oder Sonderlichen, indem sie innerhalb derselben - z.B. chemischen - Gruppe die Unterschiede unter den Arzneien ermittelten.

*Schriftliche Version des Festvortrags des Autors zum 25-jährigen Bestehen der SAHP.

\title{
Zum 25-jährigen Bestehen der Schweizerischen Ärztegesellschaft für Homöopathie SAHP*
}

Ammonium muriaticum $\left(\mathrm{NH}_{4} \mathrm{Cl}\right)$ als Beispiel zeigte - in der Arzneimittelprüfung am Gesunden - Schmerzen oder Versteifungen in allen Gelenken (Skaleninvarianz); die allgemeine Gelenkaffektion «fällt auf» und gehört somit zum Wesen dieser Arznei. Ammonium mur., das mit dem Stickstoffanteil zur Gruppe 15 des Periodensystems gehört, lässt sich nach Hering mit allen Arzneien der Gruppe 15 vergleichen, also mit Ammonium carbonicum, Nitri acidum, Phosphor, Arsen usw., allenfalls auch mit Calcium phosphoricum, aber auch mit den Chloriden, die zur Gruppe 17 gehören. So ermittelt man das Ungewöhnliche und Sonderliche.

Im Vergleich zwischen Ammonium mur. und Ammonium carb. nennt Farrington [2] folgende Gemeinsamkeiten und damit etwas Eigenheitliches: Bei beiden sind die Menses von dunklem Blut, «häufig in Form von Klumpen ... Vor den Menses Greifen und Kolik, ... Schlaflosigkeit, ... Frostigkeit ...». Bei Ammonium mur. hingegen findet sich als Sonderheit «ein spannender Schmerz in beiden Leisten, der die Patientin zwingt, krumm $\mathrm{zu}$ gehen ..., heftige Schmerzen im Kreuz, besonders nachts. Eiweissartiger, brauner und schleimiger Fluor folgt». Bei Ammonium carb. jedoch besteht ein «wässeriger, ätzender Fluor mit ... Ulzeration in der Vulva ...». Auf diese Weise werden wir dem Paragraphen 153 des Organons [1] sowohl bezüglich Patient als auch bezüglich Arznei gerecht. Das ist unsere Art, Ganzheitsmedizin zu betreiben, aber nicht nur.
Wir sehen die Homöopathie gesamthaft eingebunden in die Naturwissenschaft und im Kontext eines übergeordneten und damit deterministischen Prinzips. Hahnemann sprach in diesem Zusammenhang von Mathematik: «Was wird aber dann erst an Heilung ... ausgerichtet werden können, wenn mehrere genaue und zuverlässige Beobachter sich ... durch sorgfältige Selbstversuche verdient gemacht haben werden! Dann wird das Heilgeschäft den mathematischen Wissenschaften an Zuverlässigkeit nahe kommen» [3]. Und Hering schrieb: «Wir werden, was Hahnemann in Aussicht stellte, bis zur mathematischen Gewissheit gelangen» [4]. Diese Aussagen übrigens waren und sind noch immer die treibende Kraft zur Schaffung ver-

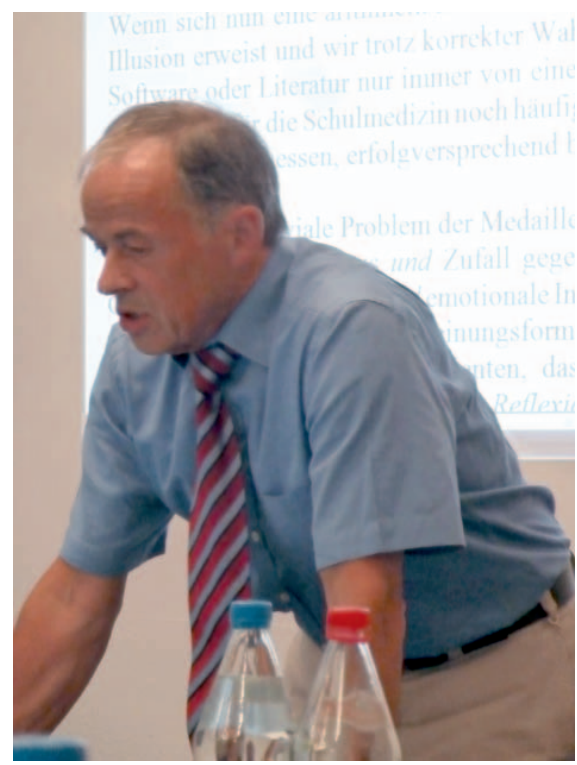

Abb. Dr. med. Urs Steiner bei seinem Festvortrag.

\section{KARGER}

Fax +497614520714 Information@Karger.d www.karger.com
(๑) 2011 S. Karger GmbH, Freiburg
Dr. med. Urs Steiner

Staldenstrasse 10, 6405 Immensee, Schweiz

dr.urs.steiner@bluewin.ch 
schiedener Softwares, z.B. von «amokoor». Es stellt sich folgende Frage: Wie weit sind wir heute - nach der Einbindung von Symptomen und Modalitäten in Gradskalen und mathematische Polaritätsanalysen - mit der Annäherung der Homöopathie an eine mathematische Sicherheit gelangt? Was bedeutet überhaupt Mathematik im Zusammenhang mit der Homöopathie?

Unter mathematischer Sicherheit verstehen wir bekanntlich in der SAHP nicht eine einfache Arithmetik, sondern wir sehen unsere Arzneimittelwahl als einen Bestandteil der nichtlinearen Dynamik, somit eingebettet in eine fraktale Welt, worin Determinismus und Zufall gleichberechtigte, ja sich bedingende Partner sind. Nur in solch einer fraktalen Welt ist es berechtigt, von «mathematischer Gewissheit» (gemeint ist Wahrscheinlichkeit) zu sprechen. Mathematische Sicherheit bedeutet in unserem Sinne die Gewissheit, dass die Komplexität eines Krankheitsgeschehens homöopathisch wohl immer und nur annäherungsweise erfasst werden kann; es bedeutet auch, die Grenzen unseres Handelns zu erkennen.

Wenn sich nun eine arithmetische Heilungs-«Gewissheit» fraktal betrachtet als eine Illusion erweist und wir trotz korrekter Wahl einer Arznei mithilfe einer sehr guten Software oder Literatur nur immer von einer fraktalen Wahrscheinlichkeit ausgehen dürfen (was für die Schulmedizin noch häufiger gälte!), wie dann aber können wir den Patienten angemessen, erfolgversprechend behandeln? Es ist das alte, triviale Problem der Medaille mit den zwei Seiten: So, wie sich in der Evolution Determinismus und Zufall gegenseitig bedingen, wie sich menschliche Grösse nur durch rationale und emotionale Intelligenz entwickelt, muss das Kranksein mit all seinen objektiven Erscheinungsformen mit dem subjektiven Emp- finden des Patienten in Einklang gebracht werden. Dies lässt sich in seiner ganzen Bedeutung nur durch die emotionale Reflexion des behandelnden Arztes erfassen, der wiederum sein Mitempfinden mit dem emotionalen Erfassen der homöopathischen Arznei in Synthese bringen muss. Dies alles - so bin ich überzeugt - wurde und wird in der SAHP stets gelehrt und gelernt. In diesem Sinne erhält die Homöopathie ihr menschliches Gesicht und damit ihre ethische Bedeutung. Wer es fassen kann ...?

\section{Literatur}

1 Hahnemann S: Organon der Heilkunst. $\$ 153$. Stuttgart, Haug, 1999.

2 Farrington EA: Vergleichende homöopathische Arzneimittellehre. Ruppichteroth, Similimum, 1995.

3 Hahnemann S: Organon der Heilkunst. $\$ 145$. Stuttgart, Haug, 1999.

4 Hering C: Medizinische Schriften in 3 Bänden. Göttingen, Ulrich Burgdorf, 1988. 